V. Tkach ${ }^{1,2}$, C. S. de Oliveira ${ }^{2}$, J. Maia ${ }^{2}$, Soares B. Gunter ${ }^{3}$, R. Ojani ${ }^{4}$, P. I. Yagodinez ${ }^{1}$

${ }^{1}$ Chernovitsky national University,

Ukraine

${ }^{2}$ Federal University of state of Mato Grosso do Sul, Brazil

${ }^{3}$ Federalny the state University of Rio de Janeiro, Brazil

${ }^{4}$ University of Mazandaran, Islamic Republic of Iran E-mail: nightwatcher2401@gmail.com

\title{
The mathematical description of the electrosynthesis of composites of oxy-hydroxycompounds cobalt with polypyrrole overooxidazed
}

The electrosynthesis of the composite with of the overoxidized polypyrrole with cobalt oxy-hydroxide in strongly acidic media has been described mathematically, using linear stability theory and bifurcation analysis. The steadystates stability conditions and oscillatory and monotonic instability requirements have been described too. The system's behavior was compared with behavior of other systems with overoxidation, electropolymerization of heterocyclic compounds and electrosynthesis of the cobalt oxy-hydroxides.

Keywords: cobalt, polypyrrole, overoxidation, oscillations, stable steady-state.

(C) Tkach V., de Oliveira C. S., Maia J., Gunter Soares B., Ojani R., Yagodinez P. I., 2016

\section{Introduction}

Electrically conducting or conducting polymers $(\mathrm{CP})$ occupy their place among the most studied materials during the last 50 years [1-4], due to their ability to combine the properties of plastics (flexibility and corrosion resistance) with metallic conductivity, so they are called synthetic metals. Another advantage that they possess is the ease of modification, giving them a wide and rich range of applications, from anticorrosive coatings to sensors and biosensors [5-12].
On the other hand cobalt is one of the most commonly used metals [13-14]. Its oxy-hydroxycompounds, in the form of films, can be used as anticorrosion coatings, as well as the surfaces for photoelectrocatalysis, due to to their intensively black color [15-16], therefore, their composites with conducting polymers should also exhibit interesting properties.

However, the oxidation of compounds of bivalent cobalt to trivalent occurs at relatively high potentials (from $+1.6 \mathrm{~V}$ ), 
in which some conducting polymers, such as polypyrrole [17-18], undergo a process
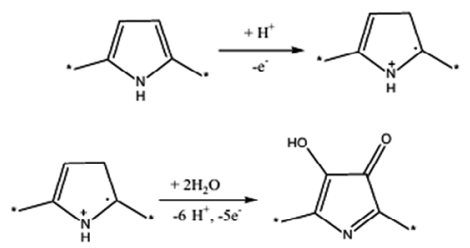

of so-called "overoxidation", known as reconfiguration of the conjugated system in the polymer according to the following mechanism.

Thus overoxidative polypyrrole conducts electricity worse than neutral. Another problem may be caused by electrochemical oscillatory and monotonic instabilities, capable to accompany the processes of electrochemical oxidation of small organic molecules (including elec-

\section{System and model}

For the mathematical description of potentiostatic electrosynthesis of the composite of the overoxidized polypyrrole with $\mathrm{CoO}(\mathrm{OH})$, from neutral polypyrrole in strongly acidic media without fluorine ions, we introduce three variables: $c$ concentration of ions of bivalent cobalt in the surface layer; $\theta$ - degree of surface filling overoxidized polypyrrole; $h$ is the concentration of protons in the surface layer.

To simplify the model, we suppose that the reactor is intensively stirring, and the supporting electrolyte is in excess, allowing neglect the influence of convective and migration flows. The thickness of the surface layer, the concentration profile of cobalt salts and protons, assumed to be linear, is constant and equal to $\delta$.

The protons diffuse in surface layer by their diffusion and are formed as a result of the process of overoxidation, their concentration in the surface layer decreases tropolymerization [19-28]), and conducting polymers [29-32]. The oscillations also accompany the process of $\mathrm{CoO}(\mathrm{OH})$ formation [15-16]. The study of their causes is one of the key stages to determine the most probable mechanism of this process.

So far, such phenomena has gained only phenomenological interpretation, which, though it may proceed from a logical conviction, has no solid theoretical justification, that can be given only by analyzing mathematical models, can adequately describe the process, which will be done in this work.

This analysis can explain the behavior of such systems and (as a consequence of the theorem of Kirpichev and Guchman) compare them with other similar systems are described, for example in [33-49].

due to proton attack of overoxidative polypyrrole. Thus, considering the first law and Fick coefficient modeling related to electric double layer (EDL), the balance equation of the concentration of protons will look like:

$$
\frac{d h}{d t}=\frac{2}{\delta}\left(\frac{D}{\delta}\left(h_{0}-h\right)+r_{o}-r_{a}\right),
$$

where $h_{0}$ is the concentration of protons in the bulk solution, $r_{o}$ is the speed of the process of overoxidation, $r_{a}$ is the rate of proton attack of overoxidative polymer.

The conventional conductive polymer, occupying in the beginning of the reaction the entire electrode, overoxidizes. Thus, overoxidated polymer interacts with the cobalt salt, forming a composite with the product of its oxidation, and is attacked by protons. Thus, the balance equation for the concentration of the overoxidative polymer can be written as 


$$
\frac{d \theta}{d t}=\frac{1}{\Gamma}\left(r_{o}-r_{a}-r_{c}\right) .
$$

In which $r_{c}$ is the reaction rate of the composite formation, and $\Gamma$ is the polymer maximum surface concentration.

The cobalt salt diffuses to the anode, and then oxidized in the overoxidative polypyrrole with the formation of the composite and the overoxidation product. Thus, the balance equation of its concentration in the subsurface layer can be written as:

$$
\frac{d c}{d c}=\frac{2}{\delta}\left(\frac{\Delta}{\delta}\left(c_{0}-c\right)-r_{c}\right),
$$

being $\mathrm{C}_{0}$ is the concentration of the cobalt salt in the solution bulk. The rate of the correspondent reactions can be expressed as:

$$
\begin{gathered}
r_{o}=k_{o}(1-\theta) h \exp \left(\frac{n_{1} F \varphi_{0}}{R T}\right) r_{a}=k_{a} \theta_{h} . \\
r_{c}=k_{c} \theta_{c} \exp \left(\frac{n_{2} F \varphi_{0}}{R T}\right),
\end{gathered}
$$

where the $k$ parameters represent rate constants of respective reactions, parameters $n$ is the number of electrons transferred in electrochemical processes, $\varphi_{0}$ is the potential drop of the EDL, in comparison with the potential of zero charge $F=N_{A} \cdot e$ is the number of Faraday.

Differential equations (1-3) resemble the systems of equations, described for the case of polypyrrole overoxidation in a strongly acidic media [33], and for the case of the electrosynthesis of oxyhydroxycompounds of cobalt [34], so it is possible to confirm the third theorem of similarity. However, in order to prove the behavior of such systems, it is necessary to compare the investigation of the mentioned systems with the system of equations (1-3), as will be illustrated below.

\section{Results and discussion}

In order to research the behavior of the system with potentiostatic electrosynthesis of composite of $\mathrm{CoO}(\mathrm{OH})$ - the overoxidative polypyrrole in strongly acidic media, it is necessary to investigate the system of differential equations (1-3), taking in account the algebraic relations (4-6), using linear stability theory. Functional Jacobi matrix, the elements of which are computed for a stationary state is represented in the form:

$$
\left(\begin{array}{lll}
a_{1_{1}} & a_{1_{2}} & a_{1_{3}} \\
a_{2_{1}} & a_{2_{2}} & a_{2_{3}} \\
a_{3_{1}} & a_{3_{2}} & a_{3_{3}}
\end{array}\right),
$$

where:

$$
a_{1_{1}}=\frac{2}{\delta}\left(-\frac{D}{\delta}+k_{o}(1-\theta) \exp \left(\frac{n_{1} F \varphi_{0}}{R T}\right)-k_{a} \theta\right) \text {. }
$$

$$
\begin{gathered}
a_{1_{2}}=\frac{2}{\delta}\left(k_{o} h \exp \left(\frac{n_{1} F \varphi_{0}}{R T}\right)+k_{o} h \theta \alpha \exp \left(\frac{n_{1} F \varphi_{0}}{R T}\right)-k_{a} h\right) . \\
a_{1_{3}}=0 . \\
a_{2_{1}}=\frac{1}{\Gamma}\left(k_{o}(1-\theta) \exp \left(\frac{n_{1} F \varphi_{0}}{R T}\right)-k_{a} \theta\right) . \\
a_{2_{2}}=\frac{1}{\Gamma}\left(k_{o} h \exp \left(\frac{n_{1} F \varphi_{0}}{R T}\right)+\right. \\
+k_{o} h \theta \alpha \exp \left(\frac{n_{1} F \varphi_{0}}{R T}\right)-k_{a} h- \\
\left.-k_{c} c \exp \left(\frac{n_{2} F \varphi_{0}}{R T}\right)-k_{c} \alpha \theta c \exp \left(\frac{n_{2} F \varphi_{0}}{R T}\right)\right) . \\
a_{2_{3}}=\frac{1}{\Gamma}\left(-k_{c} \theta \exp \left(\frac{n_{2} F \varphi_{0}}{R T}\right)\right) \\
a_{3_{1}}=0 .
\end{gathered}
$$


$a_{3_{2}}=\frac{2}{\delta}\left(-k_{c} c \exp \left(\frac{n_{2} F \varphi_{0}}{R T}\right)-k_{c} \alpha \theta c \exp \left(\frac{n_{2} F \varphi_{0}}{R T}\right)\right)$

$a_{3_{3}}=\frac{2}{\delta}\left(-k_{c} \theta \exp \left(\frac{n_{2} F \varphi_{0}}{R T}\right)-\frac{\Delta}{\delta}\right)$.

The Oscillatory instability in this system is possible, because the necessary conditions of Hopf bifurcation may be satisfied. They are performed in the case of the main diagonal of the Jacobian positive elements: In this system there are three positive elements:

$$
k_{o}(1-\theta) \exp \left(\frac{n_{1} F \varphi_{0}}{R T}\right)>0,
$$

in case of strong effects of the process of overoxidation on DEL, leading to the autocatalytic filling of the electrode surface with the overoxidated polypyrrole. This can happen if the polypyrrole derivative is a strong reducing agent and thus can form fluctuations

$$
k_{o} h \exp \left(\frac{n_{1} F \varphi_{0}}{R T}\right)>0,
$$

because of the possibility of autocatalytic formation of protons in the process of overoxidation of polypyrrole. This factor was considered as a factor self-oscillating behavior of the overoxidative polypyrrole in $[33,35-36]$, and this system it also presents.

$$
-k_{c} \alpha \theta c \exp \left(\frac{n_{2} F \varphi_{0}}{R T}\right)>0,
$$

$n$ the case of strong influences on the DEC process of oxidation of cobalt salts to hydroxy-hydroxycobalamine, which, incidentally, has already been described in $[15,16,34]$.

In this case because of the greater possibility of positive feedback, the probability of self-oscillations in this system will be higher than in case of simple potentio- static overoxidation of polypyrrole or the anodic oxidation of cobalt salts.

The stability of the stationary state we analyzed according to the criterion of Routh-Hurwitz. To avoid cumbersome expressions, we introduce new parameters so as the determinant of the Jacobian matrix can be written as:

$$
\frac{4}{\delta^{2} \Gamma}\left|\begin{array}{ccc}
-\kappa_{1}+\mathrm{X} & \Sigma & 0 \\
\mathrm{X} & \Sigma-\Sigma_{3} & -\Lambda \\
0 & -\Sigma_{3} & -\Lambda-\kappa_{2}
\end{array}\right| .
$$

Using the condition Det $J<0$, which can be derived from the criterion of Routh-Hurwitz, we obtain the condition of stability of stationary state in the form of inequalities that can be resolved relative to the diffusion coefficient of cobalt salt $\kappa_{2}$ :

$$
-\kappa_{2}<\frac{\Lambda \Sigma_{3}\left(\kappa_{1}-\mathrm{X}\right)}{\sum_{3} \kappa_{1}-\Sigma \kappa_{1}-\mathrm{X} \Sigma_{3}} .
$$

Or:

$$
\kappa_{2}>\frac{\Lambda \Sigma_{3}\left(\mathrm{X}-\kappa_{1}\right)}{\Sigma_{3} \kappa_{1}-\Sigma \kappa_{1}-\mathrm{X} \Sigma_{3}}-\Lambda .
$$

Thus, the stability of the stationary state, in this case, is supported by the more rapid diffusion of cobalt salts than protons and by high oxidation rate, which shifts the right part of inequality towards more negative values (in this case, the left part of the inequality is positive). So, at high concentrations of cobalt salts, the $\mathrm{pH}$-dependence of stability of the stationary state decreases drastically. Thus, for an optimal synthesis of the composite, it is necessary to use a concentration of cobalt salts with a small margin relative to the concentration of pyrrole and $\mathrm{pH}$, but the composite will contain a quite significant proportion of the oxy-hydroxy cobalt compound. 
The $\mathrm{pH}$-decreasing leads to an increase in the value of the fraction in the right part of the inequality and, at relatively low concentrations of cobalt salts, also reduces the stabilization trend of a stationary state, even including the system leave from the stationary state, which is displayed at the point of a monotonic instability, occurring in the condition of:

$$
\kappa_{2}>\frac{\Lambda \Sigma_{3}\left(\mathrm{X}-\kappa_{1}\right)}{\Sigma_{3} \kappa_{1}-\Sigma \kappa_{1}-\mathrm{X} \Sigma_{3}}-\Lambda,
$$

represented by a N-shaped part of the voltamperogram. In a common system with potentiostatic overoxidation of polypyrrole, contrarily to this system, it is impossible.

DEL factors also strongly affect the stability of the stationary state in this system. For example, in case of strong effects of electrochemical oxidation of cobalt salts on the structure of the DEC, the parameter $\Lambda$ will have negative values which will lead to the exit of the stationary state of the field of stability. This factor is also present in other similar systems.

The presence of fluoride ions. As known, oxy-hydroxycompound of cobalt is unstable in the presence of fluoride ions, and dissolves with the formation of the complex $\left[\mathrm{CoF}_{6}\right]^{3-}$, according to the total equation:

$$
\begin{aligned}
& \mathrm{CoO}(\mathrm{OH})+6 \mathrm{HF} \rightarrow \\
& \rightarrow \mathrm{H}_{3}\left[\mathrm{CoF}_{6}\right]+2 \mathrm{H}_{2} \mathrm{O} .
\end{aligned}
$$

In this case, the model isn't capable to describe the behavior of the composite yet. The stability of the composite in the presence of fluoride ions will be analyzed in one of our next works.

\section{Conclusions}

1. The steady-state stability for this system is maintained by relatively high $\mathrm{pH}$ and a high concentration of cobalt ions in the surface layer and common to all such systems the absence of strong effects of electrochemical processes on DEL.

2. The oscillatory behavior of this system is possible, being caused not only by the autocatalytic protons' formation, as for the general case of overoxidation of the conducting polymers, but the influence on the electrochemical reactions of DEC - overoxidation and oxidation of cobalt salts.

A monotonic instability for this system is realized at relatively low $\mathrm{pH}$ and the strong effects of electrochemical reactions on the DEC.

1. Sadki S. Scottland Ph., Brodie N., Saboraud G. The mechanisms of pyrrole electropolymerizaion. Chem. Soc. Rev. 2000;29(5):283-289.

2. J. Roncali. Conjugated poly(thiophenes): Synthesis, functionalization and applications. Chem. Rev. 1992;92(4):711-738.

3. Bodnaryuk-Lupshak N., Aksimentyeva O. I. Vopr. Khim. Khim. Techn. 1998; 50:1.

4. Yamamoto T. Molecular assembly and properties of polythiophenes. NPG Asia Materials. 2010;2(2):54-60. doi: 10.1038/asiamat.2010.37.

5. Ojani R., Raoof J. B., Ahmady A., Hosseini S. R. Casp. J. Chem. 2013;2:45.

6. de Andrade V. M. Confecção de biossensores através da imobilização de biocomponentes por eletropolimerização de pirrol. Tés. M. Eng. UFRGS., Porto Alegre, 2006.

7. Tosar Rovira J. P. Estudio de la inmovilización de oligonucleótidos a eléctrodos 
modificados de oro:polipirrol, y detección electroquímica de secuencias complementarias. Tes. Lic. Bioquím., Universidad de la República, Montevideo, 2008.

8. Ramanavicius A., Ramanaviciene A., Malinauskas A. Electrochemical sensors based on conducting polymer-polypyrrole. Electrochim. Acta. 2006;51(27):60256037. doi: 10.1016/j.electacta. 2005.11.052.

9. Singh R. P. Prospects of Organic Conducting Polymer Modified Electrodes: Enzymosensors. Int. J. Electrochem. 2012;502707. URL: http://dx.doi.org/10.1155 /2012/502707.

10. Zane D., Appetecchi G. B., Bianchini C., Passerini S., Curulli A. An impedimetric glucose biosensor based on overoxidized polypyrrole thin film. Electroanalitica. 2011;23(50):1134-1141. doi: 10.1002/elen.201000576.

11. McQuade T., Pullen A., Swager T. M. Conjugated polymer based chemical sensors. Chem. Rev. 2000;100(7):2537-2574. doi: 10.1021/cr9801014.

12. Ojani R., Raoof J. B., Rahemi V. A simple and efficient electrochemical sensor for electrocatalytic reduction of nitrite based on poly94-aminoacetanilide) film using carbon paste electrode. J. Chin. Chem. Soc. 2011;58(2):247-254.

13. Sargentelli V., Ferreira A. P. Magnetic nanoparticles: The cobalt. Ecletica Química. 2010;35(4):153-163. doi: 10.1590/S0100-46702010000400020.

14. Jamison T. F., Shambayati S., Crowe W. E., Schreiber S. L. Tandem use of cobaltmediated reactions to synthesize (+)-epoxydictymene, a diterpene containing a trans-fused 5-5ring system. J. Am. Chem. Soc. 1997;119(19):4353-4363. doi: $10.1021 /$ ja970022u.

15. Stadnik O. Diss. Kand. Chim. N. Kiev, 2011.

16. O. Stadnik et. al. Int. Electrochem. Soc. Meeting. Abstract \# 2240. - 2010.

17. Ansari R. Polypyrrole Conducting Electroactive Polymers: Synthesis and Stability Studies. E- J. Chem. 2006;3(4):186-206. LINK: http://downloads.hindawi.com/ journals/chem/2006/860413.pdf

18. Ba-Shammakh M. S., Rahman S. U., Abul-Hamayel M. A., Kahraman R. Thermal effects on the process of electropolymerization of pyrrole on mild steel. 203rd ECS Meeting, April 27-May 2, 2003, Paris, France. AC1. Organic and Bioorganic Electrochemistry General Session. No. 2468. LINK: http://www.electrochem.org/dl/ $\mathrm{ma} / 203 / \mathrm{pdfs} / 2468 . \mathrm{pdf}$

19. Liu A. S., Oliveira M. A. S. Electrodeposition of polypyrrole films on aluminum from tartrate aqueous solution. J. Braz. Chem.Soc. 2007;18(1):143-152.

20. Sazou D. The dynamical behavior of the electrochemical polymerization of indole on Fe in acetonitrile-water mixtures. Synthetic Metals. 2002;130(1):45-54. doi: 10.1016/S0379-6779(02)00110-8.

21. Das I., Goel N., Agrawal N. R., Gupta S. K. Growth patterns of dendrimers and electric potential oscillations during electropolymerization of pyrrole using monoand mixed surfactants. J. Phys. Chem.-B. 2010;114(40):12888-12896. doi: 10.1021/ jp105183q.

22. Bazzaoui M., Bazzaoui E. A., Martins L., Martins J. I. Electropolymerization of pyrrole on zinc-lead-silver alloys electrodes in acidic and neutral organic media. 
Synthetic Metals. 2002;130(1):73-83. doi: 10.1016/S0379-6779(02)00101-7.

23. Das I., Goel N., Gupta S. K., Agrawal N. R. Electropolymerization of pyrrole: Dendrimers, nano-sized patterns and oscillations in potential in presence of aromatic and aliphatic surfactants. J. Electroanal. Chem. 2012;670(1):1-10. doi: 10.1016/j. jelechem.2012.01.023.

24. Aoki K., Mukoyama I., Chen J. Compettion between polymerization and dissolution of poly(3-methylthiophene) films. Russ. J. Electrochem. 2004;40(3):280-285. doi: 10.1023/B:RUEL.0000019665.59805.4c.

25. Das I., Agrawal N. R., Ansari S. A., Gupta S. K. Pattern formation and oscillatory electropolymerization of thiophene. Ind. J. Chem.-A. 2008;47(12):1798-1803.

26. Ba-Shammakh M. S. Electropolymerization of pyrrole on mild steel for corrosion protection. Thes. Ph. D. King Fahd University of Petroleum and Minerals Dharan, Saudi Arabia, 2002.

27. Pagitsas M., Dimitra S. The improved Franck-FitzHung model for the electrodissolution of iron in sulphuric acid solutions: linear stability and bifurcation analysis. Derivation of the kinetic equations for the forced franck-FitzHung models. Electrochimica Acta. 1991;36(8):1301-1308. doi: 10.1016/0013-4686(91)80009-w.

28. Pearlstein A. J., Johnson J. A. Global and conditional stability of the steady and periodic solutions of the Franck-FitzHugh model of electrodissolution of $\mathrm{Fe}$ in $\mathrm{H}_{2} \mathrm{SO}_{4}$. J. Electrochem. Soc. 1989;136(5):1290-1299.

29. Ogüz Güler M.et. al. 7th Turkish Nanoscience and Nanotechnology Conference. 2011. P. 24.

30. Roncali J. Conjugated poly(thiophenes): Synthesis, functionalization and applications. Chem. Rev. 1992;92(4):711-738.

31. Lemos Castagno K. R. Tés. D. Sc. UFRS., Porto Alegre, 2006.

32. Mostany J., Scharifker B. R. Impedance spectroscopy of undoped, doped and overoxidazrd polypyrrole films. Synthetic Metals. 1997;87(3):179-185.

33. Tkach V., Nechyporuk V., Yagodynets' P., Rusnak I. Orbital. Elec. J. Chem. 2012;4:136.

34. Tkach V., Nechyporuk V., Slipenyuk O. Ecl. Quím. 2012;37:72.

35. Ткач В. В., да Роша Ал. М. Наук. Вісник ЧНУ. 2013;658:132.

36. Tkach V., Nechyporuk V., Yagodynets P. Electropolymerization of heterocyclic compounds. Mathematical models. Ciencia e Tecnnjlogia dos Materials. 2012;24(12):54-58.

37. Tkach V., Nechyporuk V., Yagodynets' P. Proc. Quím. 2014;8:49.

38. Tkach V., Nechyporuk V., Yagodynets' P. Hryhoryak M. Cien. Techn. Mat. 2012;24:50.

39. Tkach V., Nechyporuk V., Yagodynets' P. Tecn. Met. Mat. Min. 2013;10:249.

40. Tkach V., Nechyporuk V., Yagodynets P. Description matematica de la sintesis electroquimica de polimeros conductors en la presencia de surfactants. Avances en Química. 2013;8(1):9-15.

41. Tkach V., Nechyporuk V., Yagodynets' P. Avan. Quím. 2013;8:97. 
42. Tkach V., Nechyporuk V., Yagodynets P. The mathematical stability investigation in the electrochemical polymerization of heterocyclic compounds in the oxidants' presence. Afinidad. 2013;70(561):70-74.

43. Tkach V. V., Nechyporuk V. V., Yagodynets P. I. de Oliveira S. C., de Rocha Al. M. The Mathematical Description for the electropolymerization of electrochemical modified monomers in Galvanostatic Mode. J. of Sib. Fed. Univ. Chemistry. 2014;7(3):403. URL: http://elib.sfu-kras.ru/handle/2311/13421.

44. Tkach V., Nechyporuk V., Yagodynets' P. Anais do 10o Encontro Brasileiro sobre Adsorção, Guarujá, SP, 27-30 de abril de 2014. 122(1).

45. Tkach V., Nechyporuk V., Yagodynets' P. Med. J. Chem. 2015;3:1122.

46. Tkach V., Nechyporuk V., Yagodynets P. The mathematical stability study of the work of isomerization sensors, based on conduction polymers. Anal. Bioanal. Electrochem. 2014;6(3):273-283.

47. Tkach V., Kumara Swamy B., Ojani R., Blanes M., Yagodynets' P. Orbital Elec. J. Chem. 2015;7:1.

48. Tkach V., Ojani R., Nechyporuk V., Yagodynets P. Rev. Colomb. Cien. Quím. Farm. 2014;43:35.

49. Tkach V., Ojani R., Nechyporuk V., Yagodynets' P. Orbital. Elec. J. Chem. 2014;6:142. 
В. Ткач ${ }^{1,2}$, С. С. де Оливейра ${ }^{2}$ Ж. Майя ${ }^{2}$, Б. Гюнтер Соарес ${ }^{3}$, Р. Ожани ${ }^{4}$, П. И. Ягодинец ${ }^{1}$

${ }^{1}$ Черновицкий национальный университет, Украина

${ }^{2}$ Федеральный университет uтата Мату-Гроссу-дy-Сул,

Бразилия

${ }^{3}$ Федеральный университет итата Рио-де-Жанейро,

Бразилия

${ }^{4}$ Нниверситет Мазендерана, Истамская Республика Иран E-mail:nightwatcher2401@gmail.com

\section{Математическое описание электросинтеза композитов окси-гидроксисоединений кобальта с переокисленным полипирролом}

Электросинтез композита переокисленного полипиррола с окси-гидроксисоединениями кобальта в сильнокислой среде был описан математически, с использованием линейной теории устойчивости и бифуркационного анализа. Были описаны условия устойчивости стационарного состояния, а также автоколебательной и монотонной неустойчивости. Поведение системы было сравнено с поведением других систем с переокислением, электрополимеризацией гетероциклических соединений, а также электросинтезом окси-гидроксисоединений кобальта.

Ключевые слова: кобальт, полипиррол, переокисление, автоколебания, устойчивое стационарное состояние.

(C) Ткач В., де Оливейра С. С., Майя Ж., Гюнтер Соарес Б., Ожани Р., Ягодинец П. И., 2016

\section{Введение}

Электропроводящие или проводящие полимеры (ПП) являются одними из наиболее изучаемых материа лов на протяжении последних 50 лет [1-4], что связано с их способностью соединять в себе свойства пластмасс (гибкость и противокоррозионную устойчивость) с металлической проводимостью, благодаря чему их на- зывают синтетическими металлами. Другим их преимуществом является легкость в модификации, благодаря чему у них широкий и богатый спектр применения, начиная от противокоррозионных покрытий и заканчивая сенсорами и биосенсорами [5-12].

С другой стороны, кобальт - один из наиболее часто применяемых ме- 
таллов [13-14]. Его окси-гидроксисоединения в форме пленок могут применяться в качестве противокоррозионных покрытий, а также в качестве поверхностей-носителей для фотоэлектрокатализа, благодаря своему интенсивно черному цвету [15-16], поэтому их композиты с проводящими полимерами также должны проявлять интересные свойства.

Однако окисление соединений двухвалентного кобальта до трехвалентного происходит при довольно высоком потенциале (от +1,6 В), при котором некоторые проводящие полимеры, такие как полипиррол [17-18], проходят процесс так называемого «переокисления», то есть смены конфигурации сопряженной системы в полимере согласно следующему механизму:
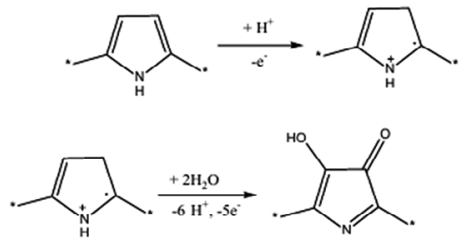

При этом переокисленный полипиррол проводит ток хуже, чем непереокисленный. Другой проблемой

\section{Система и ее модель}

Для математического описания потенциостатического электросинтеза композита переокисленного полипиррола с $\mathrm{CoO}(\mathrm{OH})$ из обычного полипиррола в сильнокислой среде без ионов фтора введем три переменные: $c$ - концентрация ионов двухвалентного кобальта в приповерхностном слое; $\theta$ - степень заполнения поверхности переокисленным полипирролом; $h$ - являются электрохимические неустойчивости - автоколебательная и (или) монотонная, которые могут сопровождать процессы электроокисления как малых органических молекул, в том числе электрополимеризацию [19-28], так и проводящих полимеров [29-32]. Автоколебания также сопровождают процесс образования $\mathrm{CoO}(\mathrm{OH})$ [1516]. Изучение их причин является одним из ключевых этапов определения наиболее вероятного механизма данного процесса.

До сих пор такие явления получали исключительно феноменологическую трактовку, которая, хоть и может исходить из логических убеждений, не имеет твердых теоретических обоснований, которые могут быть даны только с помощью анализа математической модели, способной адекватно описать процесс, что и будет показано в данной работе.

Этот анализ может объяснить поведение подобных систем (как следствие из теоремы Кирпичева - Гухмана), а также сравнить их с другими аналогичными системами, описанными, например в [33-49].

концентрация протонов в приповерхностном слое.

С целью упрощения модели мы вводим допущения того, что реактор находится при постоянном перемешивании, а фоновый электролит находится в избытке, что позволяет пренебречь влиянием конвективного и миграционного потока. При этом толщина приповерхностного слоя, концентрационный профиль солей ко- 
бальта и протонов в котором по допущению является линейным, постоянна и равна $\delta$.

Протоны попадают в диффузионный приповерхностный слой вследствие диффузии и образуются в результате процесса переокисления, их концентрация в приповерхностном слое уменьшается из-за протонной атаки переокисленного полипиррола. Таким образом, учитывая первый закон Фика и коэффициент моделирования, относящийся к двойному электрическому слою (ДЭС), уравнение баланса концентрации протонов будет выглядеть как:

$$
\frac{d h}{d t}=\frac{2}{\delta}\left(\frac{D}{\delta}\left(h_{0}-h\right)+r_{o}-r_{a}\right),
$$

где $h_{0}$ - это концентрация протонов в объеме раствора, $r_{o}$ - скорость процесса переокисления, $r_{a}-$ скорость протонной атаки переокисленного полимера.

Обычный проводящий полимер, который в начале реакции занимает весь электрод, переокисляется. При этом переокисленный полимер взаимодействует с солью кобальта, образуя композит с продуктом ее окисления, а также атакуется протонами. Таким образом, балансовое уравнение для концентрации переокисленного полимера запишется как:

$$
\frac{d \theta}{d t}=\frac{1}{\Gamma}\left(r_{o}-r_{a}-r_{c}\right) .
$$

где $r_{c}$ - реакция образования композита, а $Г$ - максимальная поверхностная концентрация полимера.
Соль кобальта диффундирует к аноду, а потом окисляется на переокисленном полипирроле с образованием его композита с продуктом переокисления. Таким образом, уравнение баланса ее концентрации в приповерхностном слое запишется как:

$$
\frac{d c}{d c}=\frac{2}{\delta}\left(\frac{\Delta}{\delta}\left(c_{0}-c\right)-r_{c}\right),
$$

где $c_{0}$ - концентрация соли кобальта в объеме раствора.

Скорости соответствующих реакций можно вычислить как:

$$
\begin{gathered}
r_{o}=k_{o}(1-\theta) h \exp \left(\frac{n_{1} F \varphi_{0}}{R T}\right) r_{a}=k_{a} \theta_{h} . \\
r_{c}=k_{c} \theta_{c} \exp \left(\frac{n_{2} F \varphi_{0}}{R T}\right),
\end{gathered}
$$

где параметры $k$ отображают константы скоростей соответствующих реакций, параметры $n$ - количество электронов, переданных в электрохимических процессах, $\varphi_{0}$ - перепад потенциала ДЭС, по сравнению с потенциалом нулевого заряда, $F=N_{A} \cdot e-$ число Фарадея.

Дифференциальные уравнения (1-3) напоминают по виду уравнения систем, описанных как для случая переокисления полипиррола в сильнокислой среде [33], так и для случая электросинтеза окси-гидроксисоединений кобальта [34], что, можно сказать, подтверждает действие третьей теоремы подобия. Однако для того, чтобы доказать подобное поведение систем, нужно сопоставить исследования указанных систем и системы уравнений (1-3), что будет показано ниже.

\section{Результаты и обсуждение}

Чтобы исследовать поведение системы с потенциостатическим элек- тросинтезом композита $\mathrm{CoO}(\mathrm{OH})$ переокисленный полипиррол в силь- 
нокислой среде, нужно разобрать систему дифференциальных уравнений (1-3) с учетом алгебраических соотношений (4-6) с помощью линейной теории устойчивости. Функциональная матрица Якоби, элементы которой вычислены для стационарного состояния представляется в виде:

$$
\left(\begin{array}{lll}
a_{1_{1}} & a_{1_{2}} & a_{1_{3}} \\
a_{2_{1}} & a_{2_{2}} & a_{2_{3}} \\
a_{3_{1}} & a_{3_{2}} & a_{3_{3}}
\end{array}\right),
$$

где

$$
\left.\begin{array}{l}
a_{1_{1}}=\frac{2}{\delta}\left(-\frac{D}{\delta}+k_{o}(1-\theta) \exp \left(\frac{n_{1} F \varphi_{0}}{R T}\right)-k_{a} \theta\right) . \\
a_{1_{2}}=\frac{2}{\delta}\left(k_{o} h \exp \left(\frac{n_{1} F \varphi_{0}}{R T}\right)+k_{o} h \theta \alpha \exp \left(\frac{n_{1} F \varphi_{0}}{R T}\right)-k_{a} h\right) . \\
a_{1_{3}}=0 . \\
a_{2_{1}}=\frac{1}{\Gamma}\left(k_{o}(1-\theta) \exp \left(\frac{n_{1} F \varphi_{0}}{R T}\right)-k_{a} \theta\right) . \\
a_{2_{2}}=\frac{1}{\Gamma}\left(k_{o} h \exp \left(\frac{n_{1} F \varphi_{0}}{R T}\right)+\right. \\
+k_{o} h \theta \alpha \exp \left(\frac{n_{1} F \varphi_{0}}{R T}\right)-k_{a} h- \\
\left.-k_{c} c \exp \left(\frac{n_{2} F \varphi_{0}}{R T}\right)-k_{c} \alpha \theta \exp \left(\frac{n_{2} F \varphi_{0}}{R T}\right)\right) . \\
a_{3_{3}}=\frac{2}{\delta}\left(-k_{c} \theta \exp \left(\frac{n_{2} F \varphi_{0}}{R T}\right)-\frac{\Delta}{\delta}\right) . \\
a_{2_{3}}=\frac{1}{\Gamma}\left(-k_{c} \theta \exp \left(\frac{n_{2} F \varphi_{0}}{R T}\right)\right) \\
a_{3_{1}}=0 . \\
\frac{2}{\delta}\left(-k_{c} c \exp \left(\frac{n_{2} F \varphi_{0}}{R T}\right)-k_{c} \alpha \theta c \exp \left(\frac{n_{2} F \varphi_{0}}{R T}\right)\right) \\
a_{3_{2}}
\end{array}\right) .
$$

\section{Автоколебательная неустойчи-} вость в данной системе возможна, так как могут выполняться необходимые условия бифуркации Хопфа. Они выполняются в случае наличия в главной диагонали якобиана положительных элементов. В данной системе есть три положительных элемента:

$$
k_{o}(1-\theta) \exp \left(\frac{n_{1} F \varphi_{0}}{R T}\right)>0,
$$

в случае сильных влияний процесса переокисления на ДЭС, приводящих к самоускоренному заполнению поверхности электрода переокисленным полипирролом. Это может произойти, если данное производное полипиррола является сильным восстановителем, и таким образом могут образовываться колебания

$$
k_{o} h \exp \left(\frac{n_{1} F \varphi_{0}}{R T}\right)>0,
$$

ввиду возможности автокаталитического образования протонов в процессе переокисления полипиррола. Этот фактор рассматривался как фактор автоколебательного поведения при переокислении полипиррола в [33, 35-36] и в данной системе он тоже присутствует.

$$
-k_{c} \alpha \theta c \exp \left(\frac{n_{2} F \varphi_{0}}{R T}\right)>0,
$$

в случае сильных влияний на ДЭС процесса окисления соли кобальта до окси-гидроксисоединения, что, кстати, уже было описано и в $[15,16,34]$.

При этом ввиду большей возможности положительной обратной связи, вероятность автоколебаний в данной системе будет выше, чем в случае простого потенциостатического переокисления полипиррола или анодного окисления соли кобальта.

Устойчивость стационарного состояния анализируем по критерию Payca - Гурвица. Для этого, чтобы 
избежать появления громоздких выражений, вводим новые параметры таким образом, что определитель матрицы Якоби запишется как:

$$
\frac{4}{\delta^{2} \Gamma}\left|\begin{array}{ccc}
-\kappa_{1}+\mathrm{X} & \Sigma & 0 \\
\mathrm{X} & \Sigma-\Sigma_{3} & -\Lambda \\
0 & -\Sigma_{3} & -\Lambda-\kappa_{2}
\end{array}\right| .
$$

Используя условие Det $J<0$, которое можно вывести из критерия Рауса Гурвица, мы получим условие устойчивости стационарного состояния в виде неравенства, которое можно разрешить относительно параметра диффузии соли кобальта $\kappa_{2}$ :

$$
-\kappa_{2}<\frac{\Lambda \Sigma_{3}\left(\kappa_{1}-X\right)}{\Sigma_{3} \kappa_{1}-\Sigma \kappa_{1}-X \Sigma_{3}} .
$$

или:

$$
\kappa_{2}>\frac{\Lambda \Sigma_{3}\left(\mathrm{X}-\kappa_{1}\right)}{\Sigma_{3} \kappa_{1}-\Sigma \kappa_{1}-\mathrm{X} \Sigma_{3}}-\Lambda .
$$

Таким образом, устойчивость стационарного состояния поддерживается более быстрой диффузией соли кобальта, чем протонов, а также высокой скоростью ее окисления, которая смещает правую часть неравенства в сторону более отрицательных значений (при этом левая часть неравенства остается положительной). При этом при высокой концентрации соли кобальта рН-зависимость устойчивости стационарного состояния резко убывает. Таким образом, для оптимального синтеза композита нужно использовать концентрацию соли кобальта с небольшим запасом относительно концентрации пиррола и $\mathrm{pH}$, однако в композите будет довольно значительная доля окси-гидроксисоединения кобальта.
Понижение рН приводит к увеличению значения дроби в правой части неравенства и, при относительно малых концентрациях соли кобальта и уменьшении вероятности стабилизации стационарного состояния, включая даже выход системы из стационарного состояния, который отображается в точке монотонной неустойчивости, условие появления которой:

$$
\kappa_{2}>\frac{\Lambda \Sigma_{3}\left(\mathrm{X}-\kappa_{1}\right)}{\Sigma_{3} \kappa_{1}-\Sigma \kappa_{1}-\mathrm{X} \Sigma_{3}}-\Lambda .
$$

Для обычной системы с потенциостатическим переокислением полипиррола, в отличие от данной системы, она невозможна.

Факторы ДЭС тоже сильно влияют на устойчивость стационарного состояния в данной системе. Например, в случае сильных влияний электроокисления соли кобальта на структуру ДЭС, параметр $\Lambda$ будет иметь отрицательные значения, которые приведут к выходу стационарного состояния из области устойчивости. Этот фактор присутствует и в других подобных системах.

Присутствие ионов фтора. Как известно, окси-гидроксисоединение кобальта неустойчиво в присутствие ионов фтора, ибо растворяется с образованием комплекса $\left[\mathrm{CoF}_{6}\right]^{3-}$ по суммарному уравнению:

$$
\begin{aligned}
& \mathrm{CoO}(\mathrm{OH})+6 \mathrm{HF} \rightarrow \\
& \rightarrow \mathrm{H}_{3}\left[\mathrm{CoF}_{6}\right]+2 \mathrm{H}_{2} \mathrm{O} .
\end{aligned}
$$

В таком случае данная модель уже не описывает поведение композита. Устойчивость композита в присутствие ионов фтора будет рассмотрена в одной из наших следующих работ. 


\section{Выводы}

Устойчивость стационарного состояния для данной системы поддерживается относительно высоким $\mathrm{pH}$ и высокой концентрацией ионов кобальта в приповерхностном слое, а также общим для всех подобных систем фактором отсутствия сильных влияний электрохимических процессов на ДЭС.

Автоколебательное поведение для данной системы является возможным, будучи вызванным не только автокаталитическим образованием протонов, как для общего случая переокисления проводящих полимеров, но и влиянием на ДЭС электрохимических реакций - переокисления и окисления соли кобальта.

Монотонная неустойчивость для данной системы реализуется при относительно низких $\mathrm{pH}$ и сильных влияниях электрохимических реакций на ДЭС.

1. Sadki S. Scottland Ph., Brodie N., Saboraud G. The mechanisms of pyrrole electropolymerizaion. Chem. Soc. Rev. 2000;29(5):283-289.

2. Roncali J. Conjugated poly(thiophenes): Synthesis, functionalization and applications. Chem. Rev. 1992;92(4):711-738.

3. Bodnaryuk-Lupshak N., Aksimentyeva O. I. Vopr. Khim. Khim. Techn. 1998;50:1.

4. Yamamoto T. Molecular assembly and properties of polythiophenes. NPG Asia Materials. 2010;2(2):54-60. doi: 10.1038/asiamat.2010.37.

5. Ojani R., Raoof J. B., Ahmady A., Hosseini S. R. Casp. J. Chem. 2013;2:45.

6. de Andrade V. M. Confecção de biossensores através da imobilização de biocomponentes por eletropolimerização de pirrol. Tésis, Porto Alegre, 2006.Tosar Rovira J. P. Estudio de la inmovilización de oligonucleótidos a eléctrodos modificados de oro:polipirrol, y detección electroquímica de secuencias complementarias. Tesis. Lic. Bioquím., Universidad de la República, Montevideo, 2008.

7. Tosar Rovira J. P. Estudio de la inmovilización de oligonucleótidos a eléctrodos modificados de oro: polipirrol, y detección electroquímica de secuencias complementarias. Tesis. Lic. Bioquím., Universidad de la República, Montevideo, 2008.

8. Ramanavicius A., Ramanaviciene A., Malinauskas A. Electrochemical sensors based on conducting polymer-polypyrrole. Electrochim. Acta. 2006;51(27):60256037. doi: 10.1016/j.electacta. 2005.11.052.

9. Singh R. P. Prospects of Organic Conducting Polymer Modified Electrodes: Enzymosensors. Int. J. Electrochem. 2012;502707. URL: http://dx.doi. org/10.1155/2012/502707.

10. Zane D., Appetecchi G. B., Bianchini C., Passerini S., Curulli A. An impedimetric glucose biosensor based on overoxidized polypyrrole thin film. Electroanalitica. 2011;23(5):1134-1141. doi: 10.1002/elen.201000576.

11. McQuade T., Pullen A., Swager T. M. Conjugated polymer based chemical sensors. Chem. Rev. 2000;100(7):2537-2574. doi: 10.1021/cr9801014.

12. Ojani R., Raoof J. B., Rahemi V. A simple and efficient electrochemical sensor for electrocatalytic reduction of nitrite based on poly94-aminoacetanilide) film using carbon paste electrode. J. Chin. Chem. Soc. 2011;58(2):247-254. 
13. Sargentelli V., Ferreira A. P. Magnetic nanoparticles: The cobalt. Ecletica Química. 2010;35(4):153-163. doi: 10.1590/S0100-46702010000400020.

14. Jamison T. F., Shambayati S., Crowe W. E., Schreiber S. L. Tandem use of cobalt-mediated reactions to synthesize (+)-epoxydictymene, a diterpene containing a trans-fused 5-5ring system. J. Am. Chem. Soc. 1997;119(19):4353-4363. doi: 10.1021/ja970022u.

15. Stadnik O. Ph.D. Thesis. Kiev. 2011.

16. Stadnik O. et al. Int. Electrochem. Soc. Meeting. Abstract \# 2240, 2010.

17. Ansari R. Polypyrrole Conducting Electroactive Polymers: Synthesis and Stability Studies. E-J. Chem. 2006;3(4):186-206. LINK: http://downloads.hindawi.com/journals/chem/2006/860413.pdf

18. Ba-Shammakh M. S., Rahman S. U., Abul-Hamayel M. A., Kahraman R. Thermal effects on the process of electropolymerization of pyrrole on mild steel. 203rd ECS Meeting, April 27-May 2, 2003, Paris, France. AC1. Organic and Bioorganic Electrochemistry General Session. No. 2468. LINK: http://www.electrochem.org/dl/ $\mathrm{ma} / 203 / \mathrm{pdfs} / 2468 . \mathrm{pdf}$

19. Liu A. S., Oliveira M. A. S. Electrodeposition of polypyrrole films on aluminum from tartrate aqueous solution. J. Braz. Chem. Soc. 2007;18(1):143-152.

20. Sazou D. The dynamical behavior of the electrochemical polymerization of indole on Fe in acetonitrile-water mixtures. Synthetic Metals. 2002;130(1):45-54. doi: 10.1016/S0379-6779(02)00110-8.

21. Das I., Goel N., Agrawal N. R., Gupta S. K. Growth patterns of dendrimers and electric potential oscillations during electropolymerization of pyrrole using mono- and mixed surfactants. J. Phys. Chem.-B. 2010;114(40):12888-12896. doi: 10.1021/jp105183q.

22. Bazzaoui M., Bazzaoui E. A., Martins L., Martins J. I. Electropolymerization of pyrrole on zinc-lead-silver alloys electrodes in acidic and neutral organic media. Synthetic Metals. 2002;130(1):73-83. doi: 10.1016/S0379-6779(02)00101-7.

23. Das I., Goel N., Gupta S. K., Agrawal N. R. Electropolymerization of pyrrole: Dendrimers, nano-sized patterns and oscillations in potential in presence of aromatic and aliphatic surfactants. J. Electroanal. Chem. 2012;670(1):1-10. doi: 10.1016/j. jelechem.2012.01.023.

24. Aoki K., Mukoyama I., Chen J. Compettion between polymerization and dissolution of poly(3-methylthiophene) films. Russ. J. Electrochem. 2004;40(3):280-285. doi: 10.1023/B:RUEL.0000019665.59805.4c.

25. Das I., Agrawal N. R., Ansari S. A., Gupta S. K. Pattern formation and oscillatory electropolymerization of thiophene. Ind. J. Chem.-A. 2008;47(12):1798-1803.

26. Ba-Shammakh M. S. Electropolymerization of pyrrole on mild steel for corrosion protection. Thes. Ph. D. King Fahd University of Petroleum and Minerals. Dharan, Saudi Arabia, 2002.

27. Pagitsas M., Dimitra S. The improved Franck-FitzHung model for the electrodissolution of iron in sulphuric acid solutions: linear stability and bifurcation analysis. Derivation of the kinetic equations for the forced franck-FitzHung models. Electrochimica Acta. 1991;36(8):1301-1308. doi: 10.1016/0013-4686(91)80009-w. 
28. Pearlstein A. J., Johnson J. A. Global and conditional stability of the steady and periodic solutions of the Franck-FitzHugh model of electrodissolution of Fe in $\mathrm{H}_{2} \mathrm{SO}_{4}$. J. Electrochem. Soc. 1989;136(5):1290-1299.

29. Ogüz Güler M. et al. 7th Turkish Nanoscience and Nanotechnology Conference, 2011, 24.

30. Roncali J. Conjugated poly(thiophenes): Synthesis, functionalization and applications. Chem. Rev. 1992;92(4):711-738.

31. Lemos Castagno K. R. Tés. D. Sc. UFRS., Porto Alegre, 2006.

32. Mostany J., Scharifker B. R. Impedance spectroscopy of undoped, doped and overoxidazrd polypyrrole films. Synthetic Metals. 1997;87(3):179-185.

33. Tkach V., Nechyporuk V., Yagodynets P.,' Rusnak P. I. Orbital. Elec. J. Chem., 2012;4:136.

34. Tkach V., Nechyporuk V., Slipenyuk O. Ecl. Quím. 2012;37:72.

35. Ткач В. В., да Роша Ал. М. Наук. Вісник ЧНУ. 2013;658:132.

36. Tkach V., Nechyporuk V., Yagodynets P. Electropolymerization of heterocyclic compounds. Mathematical models. Ciencia e Tecnnjlogia dos Materials. 2012;24(1-2):54-58.

37. Tkach V., Nechyporuk V., Yagodynets' P. Proc. Quím. 2014;8:49

38. Tkach V., Nechyporuk V., Yagodynets' P., Hryhoryak M. The investigation of electrochemical behavior during the electroreductive synthesis of conductive polymers in presence of metal cations. Cien. Tecn. Mat. 2012;24(1-2):50-53.

39. Tkach V., Nechyporuk V., Yagodynets P.. Tecn. Met. Mat. Min. 2013;10:249

40. Tkach V., Nechyporuk V., Yagodynets P. Description matematica de la sintesis electroquimica de polimeros conductors en la presencia de surfactants. Avances en Química. 2013;8(1):9-15.

41. Tkach V., Nechyporuk V., Yagodynets' P. Avan. Quím. 2013;8:97.

42. Tkach V., Nechyporuk V., Yagodynets P. The mathematical stability investigation in the electrochemical polymerization of heterocyclic compounds in the oxidants' presence. Afinidad. 2013;70(561):70-74.

43. Ткач В. В., Нечипорук В. В., Ягодинец П. И., де Оливейра С. С., да Роша А. М. Математическое описание электрополимеризации электрохимически модифицированных мономеров в гальваностатистическом режиме. Журн. Сиб. бед. ун-та. Химия. 2014;7(3):403. URL: http://elib.sfu-kras.ru/handle/2311/13421.

44. Tkach V., Nechyporuk V., Yagodynets' P. Anais do 10o Encontro Brasileiro sobre Adsorção, Guarujá, SP, 27-30 de abril de 2014. N. 122-1

45. Tkach V., Nechyporuk V., Yagodynets' P. Med. J. Chem. 2015;3:1122.

46. Tkach V., Nechyporuk V., Yagodynets P. The mathematical stability study of the work of isomerization sensors, based on conduction polymers. Anal. Bioanal. Electrochem. 2014;6(3):273-283.

47. Tkach V., Kumara Swamy B., Ojani R., Blanes M., Yagodynets' P. Orbital Elec. J. Chem. 2015;7:1.

48. Tkach V., Ojani R., Nechyporuk V., Yagodynets P. Rev. Colomb. Cien. Quím. Farm. 2014;43:35.

49. Tkach V., Ojani R., Nechyporuk V., Yagodynets' P. Orbital. Elec. J. Chem. 2014;6:142. 